Trauma Berufskrankh 2006 • 8 [Suppl 1]: S31-S37 DOI 10.1007/s10039-006-1133-z

Online publiziert: 12. Mai 2006

(c) Springer Medizin Verlag 2006

\section{G. Germann · C. Heitmann}

Klinik für Hand-, Plastische und Rekonstruktive Chirurgie,

Schwerbrandverletztenzentrum, BG-Unfallklinik Ludwigshafen, Plastische

und Handchirurgie, Universität Heidelberg, Ludwigshafen

\title{
Ersatzoperationen bei motorischen und sensiblen Ausfällen der oberen Extremität
}

Motorische Ersatzoperationen dienen der Wiedererlangung von Muskelfunktionen, die durch Nervenlähmungen infolge Trauma oder Erkrankung oder durch direkte Muskelzerstörung verloren gegangen sind.

Trotz verbesserter mikrochirurgischer Nervennahttechniken kommt es nach peripheren Nervenverletzungen und traumatischen Plexusläsionen noch immer zu Defektheilungen mit erheblichen motorischen Ausfällen. Daraus resultieren

- Funktionsbeeinträchtigungen,

- eine deutlich verlängerte Rekonvaleszenzphase,

- eine verzögerte soziale und berufliche Reintegration und nicht zuletzt

- eine signifikante Erhöhung der Therapiekosten $[3,12,20]$.

Motorische Ersatzoperationen können in jeder Phase nach der Verletzung zu einer deutlichen Verbesserung der durch die Lähmung eingeschränkten Handfunktion führen und damit die Rehabilitationsperiode entscheidend verkürzen. Sie können bei bestimmten Verletzungen unmittelbar nach der Nervenläsion indiziert sein (s. unten), aber auch bei lange bestehenden Paresen kann mit einer motorischen Ersatzoperation eine für den Patienten bedeutsame Funktionsverbesserung erzielt werden [22].

Das Operationsprinzip beruht beim motorischen Ersatz auf der Umlagerung der Sehnen intakter Muskeln auf die Sehnen gelähmter oder geschädigter Muskeln, um deren Funktion zu übernehmen bzw. zu unterstützen. Bei sensiblen Ersatzoperationen geht es darum, innervierte Areale der Hand gefäßgestielt so umzulagern, das taktil wichtige Kontaktzonen der Hand wieder für den Greifprozess eingesetzt werden können.

Aus der Vielzahl der beschriebenen Operationsverfahren soll in der Folge eine Auswahl von Standardverfahren erläutert werden, mit deren Hilfe betroffenen Patienten motorische und sensible Funktionsverbesserungen nach typischen Nervenausfällen als therapeutische Alternative zur langfristigen Schienenbehandlung oder der dauerhaften Lähmung angeboten werden können.

\section{Parese des N. ulnaris}

\section{Ausfallmuster und Indikation für Ulnarisersatzoperationen}

Nach persistierender Schädigung des N. ulnaris stehen 2 Hauptprobleme im Vordergrund der Überlegungen zur Planung rekonstruktiver Maßnahmen [3, 12, 24, 29] :

1. Führende Indikation ist zumeist die Krallenfehlstellung (• Abb. 1a) von Ring- und Kleinfinger. Durch die Lähmung der Mm. lumbricales kommt es zur Hyperextension der
Grundgelenke und einer Beugung der Mittel- und Endgelenke, sodass die Finger ein zu greifendes Objekt bereits eingerollt erreichen.

2. Eine seltenere Indikation ist die Wiederherstellung der Abduktion des Zeigefingers und der Adduktion des Daumens zur Verbesserung des Spitzgriffs.

3. Ausfall der tiefen ulnarseitigen Beuger

4. Sensible Ausfälle führen zu einer Sensibilitätsminderung bzw. -ausfall im ulnaren Bereich des Ringfingers und am Kleinfinger und stellen normalerweise keine Indikation für rekonstruktive Maßnahmen dar.

\section{Lassooperation nach Zancolli zur Korrektur der Krallenstellung}

Über eine quere Hautinzision in Höhe der distalen Hohlhandbeugefalte werden die Beugesehnenscheiden distal des A2-Ringbandes L-förmig eröffnet und die Sehnen der Mm. flexores digitorum superficiales (im Allgemeinen FDS IV und V) etwa $1 \mathrm{~cm}$ proximal ihres Ansatzes durchtrennt $[5,30]$.

Das proximale Sehnenende wird lassoartig um das A1-Ringband herumgezogen, bis das MP-Gelenk in einer Beugung von $60^{\circ}$ steht, und im Mittelhandbereich proximal des A1-Ringbandes mit sich selbst vernäht. Dadurch wird die zur 


\section{Nervenverletzungen}
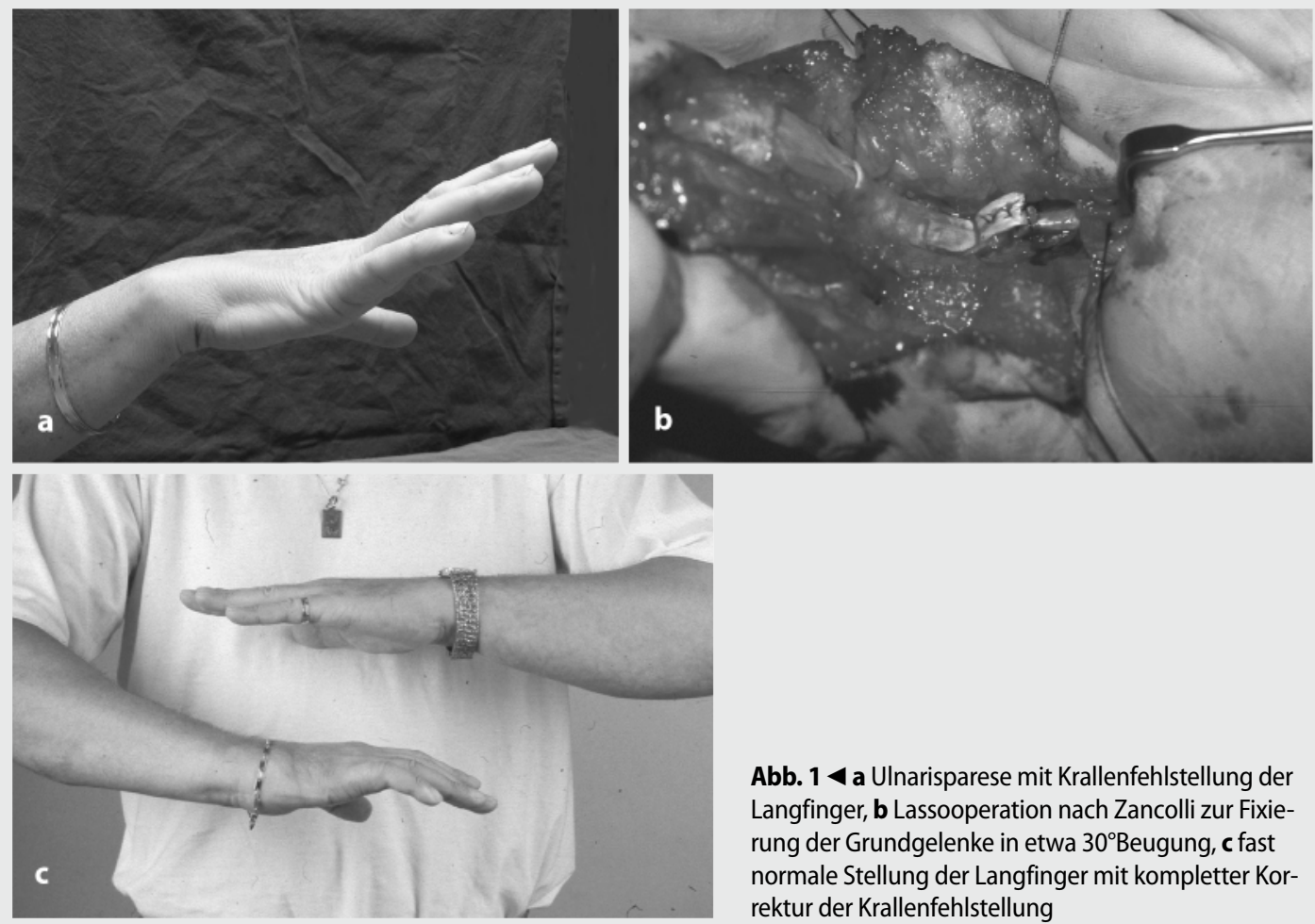

Abb. $1<$ a Ulnarisparese mit Krallenfehlstellung der Langfinger, b Lassooperation nach Zancolli zur Fixierung der Grundgelenke in etwa $30^{\circ}$ Beugung, c fast normale Stellung der Langfinger mit kompletter Korrektur der Krallenfehlstellung

Vermeidung einer erneuten Hyperextension notwendige Beugung des Grundgelenks erhalten. Alternativ kann auch die Sehne des M. flexor digitorum superficialis IV in 2 Teile aufgespalten und für beide Finger benutzt werden [6] (• Abb. 1).

\section{Statische MP-Fixation}

Sie ist eine Alternative zur Lassooperation. Im Bereich des A1-Ringbandes wird die palmare Platte des MP-Gelenks dargestellt, mobilisiert und proximalisiert, wodurch das MP-Gelenk in Beugung gebracht wird. Die Fixation erfolgt entweder durch durchgreifende Periostnähte oder intraossäre Knochenanker [30].

Nachbehandlung/Prognose. Für 6 Wochen wird eine dorsale Unterarmgipsschiene (Handgelenk $0^{\circ}$, MP-Gelenk $60^{\circ}$ Flexion, PIP und DIP zu $0^{\circ}$ streckbar) angelegt, danach erfolgt der schrittweise Belastungsaufbau.

Die Prognose ist bei guten Voraussetzungen, korrekt durchgeführter Technik und einwandreicher Nachbehandlung sehr gut. Die Patienten erreichen ein erstaunliches Funktionsausmaß, die ursprünglich bestehende Behinderung ist kaum erkennbar.

\section{Wiederherstellung des Spitzgriffs}

Zur Rekonstruktion der Kraft des Spitzgriffs müssen die Stabilität im Grundgelenk wiederhergestellt, die Flexion im Endgelenk bei der Durchführung des Schlüsselgriffs verhindert sowie Adduktion und Retropulsion verbessert werden. Zur Sehnentransposition stehen dabei verschiedene Methoden zur Verfügung $[4,13,19]$.

Rekonstruktion der Daumenadduktion. Die Sehne des M. flexor superficialis IV kann um prätendinöse Anteile der Palmaraponeurose und von dort entweder subkutan zum radialen Sesambein am Daumengrundgelenk oder zur Sehne des M. adductor pollicis geleitet werden.

Alternativ kann neben der Sehne des M. extensor indicis proprius zur Adduktionsplastik auch die des M. extensor carpi radialis brevis verwandt werden. Letztere wird nach palmar zwischen $\mathrm{MHK}_{3}$ und MHK 4 umgeleitet und an der Adduktoraponeurose fixiert, wobei wegen fehlender Länge der Spendersehne zumeist ein freies Sehnentransplantat erforderlich ist.
Verbesserung der Radialabduktion des Zeigefingers. Sie geschieht am einfachsten durch Transposition der Sehne des M. extensor indicis proprius auf distale Sehnenanteile des M. interosseus I. Dafür wird die Sehne des M. extensor indicis proprius abgetrennt und am Handgelenk hervorgezogen. Anschließend erfolgt die subkutane Tunnelung zwischen Mittelhandknochen I und II zur Insertion des 1. dorsalen $M$. interosseus an der Radialseite des MP-Gelenks des Zeigefingers, wobei palmar der Gelenkachse in straffer Spannung fixiert wird, sodass der Zeigefinger in eine leichte Grundgelenkbeugung sowie Radialabduktion kommt.

Nachbehandlung/Prognose. Nach Tragen einer dorsalen Gipsschiene für 3 Wochen wird mit Koordinations- und Greifübungen aus der Schiene heraus begonnen.

Die Prognose ist gut [12].

\section{Ersatz der tiefen ulnaren Beuger}

Hier bieten sich die Koppelung der Sehnen der gelähmten Muskulatur mit N.medianus-innervierten Beugern an, sodass synergistische Bewegungsmuster vorliegen. 


\section{Parese des N. radialis}

\section{Ausfallmuster und Indikation für Radialisersatzoperationen}

Die proximale (hohe) Radialisparese mit kompletter Fallhand tritt nach Verletzung des N. radialis durch Humerusschaftfrakturen oder nach operativer Versorgung proximaler Radiusfrakturen auf. Davon abzugrenzen ist die Lähmung des überwiegend motorischen tiefen Asts, der zum Ausfall aller Fingerstrecker, des M. extensor pollicis longus und des M. extensor carpi ulnaris führt. Die Handgelenkstreckung bleibt dabei erhalten, es resultiert aber eine deutliche Deviation der Hand nach radial, da beide ECR-Muskeln erhalten sind. Sensible Ausfälle führen zu einer begrenzten Sensibilitätsminderung bzw. -ausfall im Bereich des Unterarms und stellen keine Indikation für rekonstruktive Maßnahmen dar.

Sowohl die primäre Nervennaht als die auch die Nervenrekonstruktion mit Transplantaten haben beim N. radialis eine fragliche Prognose [1]. Entscheidet man sich für eine abwartende Haltung, geht dies häufig mit einer Schienenbehandlung von mehr als 1 Jahr für den Patienten einher, ohne dass eine befriedigende Funktionsrückkehr garantiert ist. Je nach persönlichem Profil des Patienten und der Chance auf Regeneration des rekonstruierten Nervs bieten wir heute eine Ersatzoperation in den ersten 6 Wochen nach dem Unfall an. Dies erlaubt eine Rückkehr in die berufliche Tätigkeit innerhalb von 6-12 Wochen postoperativ. Je nach Ausfallsmuster kommen hier$\mathrm{zu}$ augmentierende oder komplett ersetzende Verfahren zum Einsatz.

\section{Radialisersatzplastiken}

Unter zahlreichen beschriebenen Varianten ist die Radialisersatzoperation nach Merle d'Aubigné die wohl am häufigsten gebrauchte Grundtechnik. Bei ihr werden die Sehne des M. flexor carpi ulnaris auf die Sehnen des M. extensor digitorum communis und des M. extensor pollicis longus, die Sehne des M. pronator teres auf die Sehnen der Mm. extensor carpi radialis longus und brevis sowie die Sehne des M. palmaris longus auf

Trauma Berufskrankh 2006 - 8 [Suppl 1]: S31-S37 DOI 10.1007/s10039-006-1133-z

(c) Springer Medizin Verlag 2006

\section{G. Germann · C. Heitmann}

\section{Ersatzoperationen bei motorischen und sensiblen} Ausfällen der oberen Extremität

\section{Zusammenfassung}

Nervenverletzungen der oberen Extremität nach Trauma oder Tumorexzision führen meist zu schweren Funktionsbeeinträchtigungen. Betroffen sind überwiegend aktive Erwachsene, sodass neben der individuellen Behinderung auch die sozioökonomischen Auswirkungen beachtlich sind. Bei günstigen Ausgangskriterien ist eine Nervenrekonstruktion gegenüber einer Ersatzoperation zu bevorzugen, da Letztere immer eine Hebemorbidität bedingt. Die Prognose peripherer Stammnervenverletzungen ist unterschiedlich. Die hohe Radialisparese zeigt auch nach primärer Rekonstruktion oft unbefriedigende Ergebnisse, v. a. bei älteren Patienten, bei welchen die Regenerationsprozesse nicht mehr in gleicher Qualität ablaufen wie bei Jüngeren. Motorische und sensible Ersatzoperationen können in jeder Phase nach der Verletzung zu einer Wiederherstel-

lung verlorener Nervenfunktionen und damit einer deutlichen Verbesserung der durch die Lähmung eingeschränkten Handfunktion führen. Damit besteht eine therapeutische Alternative zur dauerhaften Lähmung bzw. langfristigen Schienenbehandlung, und die Rehabilitationsperiode kann entscheidend verkürzt werden. Differenzierte Techniken vermindern die Hebedefektmorbidität, auch bestehen keine Koordinationsprobleme hinsichtlich der rekonstruierten Nerven. Die häufigsten motorischen und sensiblen Ersatzoperationen werden vorgestellt, was künftige klinische Indikationsstellungen erleichtern soll.

\section{Schlüsselwörter}

Nervenlähmung · Obere Extremität · Motorische Funktion · Sensibilität · Nervenrekonstruktion

\section{Nerve repair after loss of motor function and sensibility of the upper extremity}

\section{Abstract}

Nerve injuries in the upper extremity after trauma or tumor resection can result in severe disability. Patients are usually adults with a high activity level, so these injuries not only impact their quality of life but also significantly affect their professional reintegration. In the past three decades, progress in microsurgical techniques and instruments has resulted in improved outcomes for nerve injuries. If the prognosis for nerve repair is reasonably good, it is usually preferred over tendon or flap transfer procedures. However, lesions of peripheral nerves, such as a high radial nerve palsy, may still yield unsatisfactory results. The prognosis also depends on the patient's age, as the regenerative pro- cess is delayed in older patients. Tendon or flap transfers to restore sensation can be performed at any time posttrauma. These procedures abbreviate the time when hand function is severely impaired so that the patient has alternatives to persistent nerve palsy with the necessity for permanent external splints. This paper gives an overview of the most common procedures for restoring hand function and sensation in the most important digits, and it provides help for decision making.

\section{Keywords}

Nerve palsy · Upper limb · Motor function . Tendon transfer $\cdot$ Restoration of sensibility 

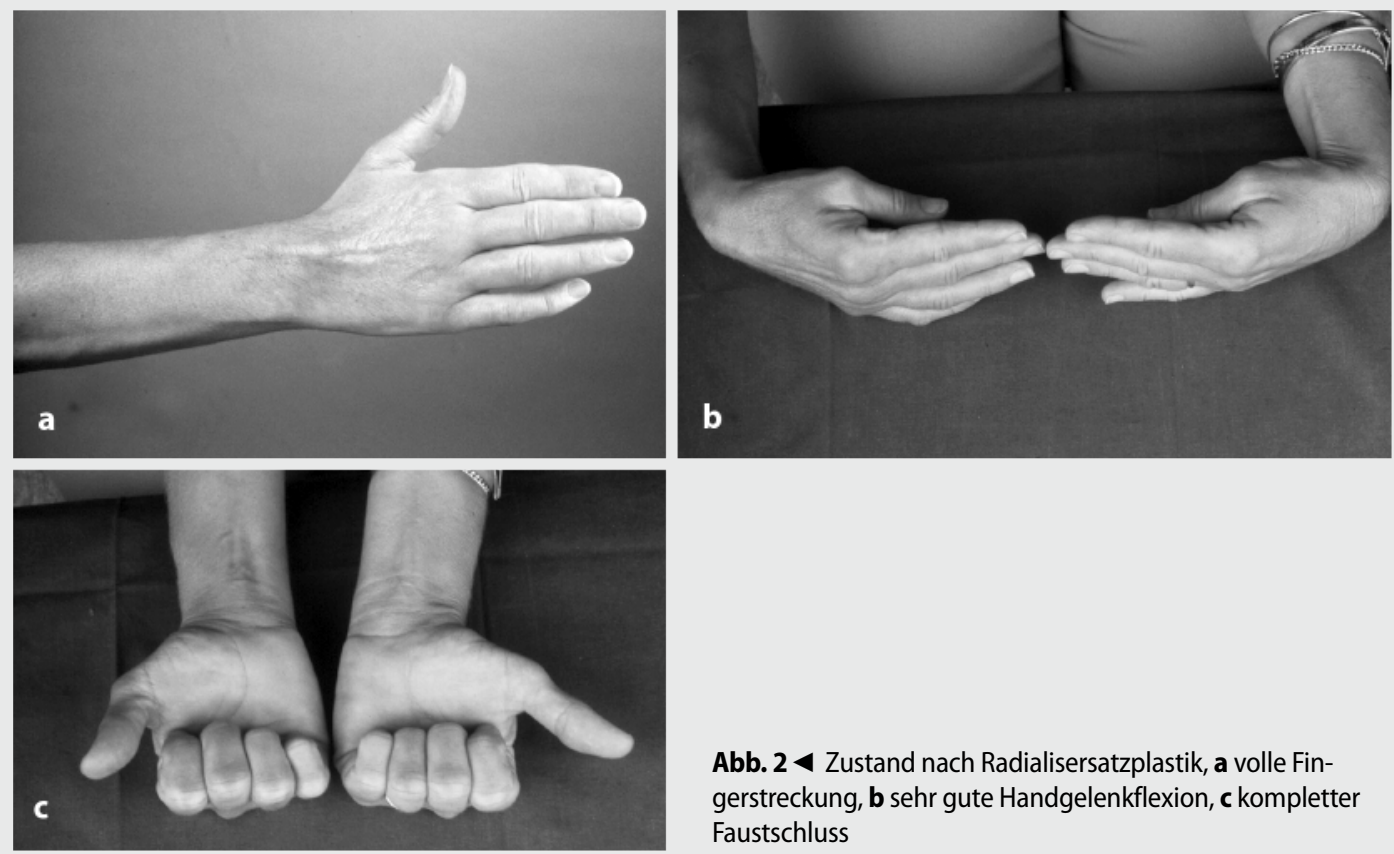

Abb. 24 Zustand nach Radialisersatzplastik, a volle Fingerstreckung, b sehr gute Handgelenkflexion, c kompletter Faustschluss

die Sehnen des M. extensor pollicis brevis und des M. abductor pollicis longus umgelagert. Dies erfolgt durch Einflechtung in die intakt belassenen Originalsehnen in durchflechtender End-zu-End-PulvertaftTechnik. Bei Fehlen des M. palmaris kann alternativ auf die Sehnen oberflächlicher Beuger $[\mathrm{Mm}$. flexores digitorum superficialis III und IV (Technik nach Boyes)] zurückgegriffen werden [23].

Nach erfolgter Naht sollte das Handgelenk passiv so beweglich sein, dass der Tenodesetest erfolgen kann: Bei Beugung müssen die Finger in ausreichende Streckung kommen. Sie sollten passiv so beweglich bleiben, dass sie ohne große Mühe in die Hand gebeugt werden können (- Abb. 2).

Partielle Radialisersatzplastik. Bei der partiellen Lähmung (Läsion des R. profundus) wählt man ein identisches Vorgehen, nur entfällt hierbei die Wiederherstellung der Handgelenkstreckung.

Nachbehandlung/Prognose. Die Ruhigstellung erfolgt für 3 Wochen mit einer palmaren Unterarmfingergipsschiene mit Daumeneinschluss, die das Handgelenk in $30^{\circ}$ Streckung, die MP-Gelenke in maximaler (Hyper)extension, PIP und DIP in Extension, den Daumen in maximaler Abduktion und Extension fixiert. Anschließend wird für weitere 3 Wochen eine palmare Schiene mit gleicher Gelenkstellung angepasst und mit Krankengymnastik aus der Schiene heraus durch erfahrene Therapeuten begonnen. Nach Freigabe der Bewegung benötigen die Patienten in der Regel noch 6-8 Wochen, bis die Hand für die Aktivitäten des täglichen Lebens eingesetzt werden kann.

Bei guten physiologischen Voraussetzungen der Spendermuskeln, aktiver Mitarbeit der Patienten und technisch einwandfreier Durchführung hat die Radialisersatzplastik eine exzellente Prognose. Viele Patienten können nach 4-5 Monaten wieder in ihre alte berufliche Tätigkeit zurückkehren [10, 18, 25].

\section{Parese des N. medianus}

\section{Ausfallmuster und Indikation für Medianusersatzoperationen}

Auch bei Paresen des N. medianus wird zwischen tiefen distalen und hohen proximalen Ausfällen unterschieden. Bei proximalen Läsionen müssen zusätzlich zur Daumenballenmuskulatur die Funktionen des langen Daumenbeugers und der tiefen Beugesehnen II und III ersetzt werden.

Sensible Ausfälle führen zu einer Sensibilitätsminderung bzw. -ausfall im Bereich von Daumen, Zeige-, Mittel- und der Radialseite des Ringfingers. Aufgrund der herausragenden Bedeutung der sensibel vom N. medianus versorgten Finger für die taktile Funktion stellt dieser Ausfall eine Indikation für rekonstruktive Maßnahmen dar.

\section{Ersatzoperation bei hoher proximaler Medianusparese}

Hier besteht die Indikation für den Ersatz sowohl der Opposition (Techniken wie unten beschrieben) als auch der Funktion des M. flexor pollicis longus und der $\mathrm{Mm}$. flexores digitorum profundus II und III. Bei Erwachsenen kann die Indikation frühzeitig gestellt werden, v. a. wenn die Aussicht auf eine befriedigende Reinnervation nach hoher Läsion fraglich ist. Für diesen Fall wird die Nervennaht mit diesem Eingriff kombiniert [3].

Neben einer Opponensplastik (s. unten) mit der Sehne des M. extensor indicis proprius wird die Sehne des M. brachioradialis auf die Sehne des gelähmten M. flexor pollicis longus, die Sehne des M. extensor carpi radialis longus auf die Sehnen der gelähmten tiefen Beugemuskeln von Zeige- und Mittelfinger verlagert. Die Verwendung dieser Muskeln ist nicht unproblematisch, da sie im Vergleich zu den ausgefallenen Beugemuskeln oder den sonst verwendeten Streckern eine deutlich kleinere Gleitamplitude und Kraftentwicklung aufweisen [7]. 
Nachbehandlung/Prognose. Nach Transposition von M. brachioradialis und M. extensor carpi radialis longus wird zuerst für 3 Wochen mittels Oberarmgipsschiene, anschließend für weitere 3 Wochen mittels dorsoradialer Unterarmgipsschiene ruhig gestellt.

Die isolierte Wiederherstellung der Beugefunktion des Zeigefingers wird durch den Transfer der Sehne des M. extensor carpi radialis longus auf die tiefe Zeigefingerbeugesehne erreicht. Dabei wird die durch einen subkutanen Tunnel über den M. brachioradialis geführte Sehne des M. extensor carpi radialis longus in die intakt belassene tiefe Zeigefingerbeugesehne eingewoben. Der Zeigefinger muss da$\mathrm{zu}$ in maximale Streckung, das Handgelenk in $30^{\circ}$ Beugung gebracht werden, die Nachbehandlung erfolgt dynamisch über 5 Wochen.

\section{Ersatzoperation bei distaler Medianusparese}

Die Parese der Thenarmuskulatur tritt häufig bei fortgeschrittenem Karpaltunnelsyndrom oder durch unzureichende Reinnervation nach Nervenrekonstruktion auf. Auch können Destruktion oder kongenitale Aplasie der Daumenballenmuskulatur die Indikation für eine muskuläre Ersatzoperation stellen.

Im folgenden Abschnitt werden 2 zur Wiederherstellung der palmaren Abduktion und/oder Opposition (Opponensplastik) geeignete Operationstechniken vorgestellt.

\section{Opponensplastik mit M. flexor digito-} rum superficialis IV. Die klassische Opponensplastik wurde bereits 1924 von Bunnell [7] angewandt. Der M. flexor digitorum superficialis hat die Vorteile ausreichender Kraft und Amplitude, von mehr als der benötigten Länge und ist ein unabhängiger, willkürlich isoliert beweglicher Muskel. Ein Nachteil liegt in der gelegentlich auftretenden Beugekontraktur des Ringfingermittelgelenks nach Sehnenentnahme [14].

In der Modifikation der Technik von Bunnell werden die Superficialiszügel des Ringfingers mindestens $1 \mathrm{~cm}$ proximal ihrer Insertion abgetrennt, um so der Ausbildung einer Schwanenhalsdeformi- tät vorzubeugen. Auch die Superficialisschlinge, durch die die Profundussehne hindurchtritt, wird durchtrennt.

Die Sehne des M. flexor digitorum superficialis wird unter der Sehne des M. flexor carpi ulnaris ulnar und durch einen subkutanen Tunnel in Richtung des radialen Daumengrundgelenks geführt und distal in die Sehne des M. abductor pollicis brevis eingeflochten. Die Sehne des M. flexor carpi ulnaris und das Os pisiforme wirken dabei als kraftförderndes Hypomochlion.

Opponensplastik mit M. extensor indicis. Dieses Verfahren ist v. a. bei Schädigung der beugeseitigen Kraftspender indiziert, wie sie durch handgelenknahe Verletzungen eintreten, die auch den N. medianus betreffen [8]. Die Abtrennung und Mobilisierung der Sehne des M. extensor indicis erfolgt in gleicher Weise wie bei der Umlagerung des M. extensor indicis zur Rekonstruktion der Daumenstreckung. Die distale Abtrennung muss allerdings $2 \mathrm{~cm}$ weiter distal unter Mitnahme eines Streifens der Aponeurose erfolgen, da ansonsten die Sehnenlänge oft nicht ausreicht.

Nach Hervorziehen der Sehne des M. extensor indicis am Handgelenk wird diese um die ulnare Handkante in Richtung Insertionsstelle am Daumen geführt. Hier erfolgt die distale Insertion entweder an der Opponenssehne oder radialseitig am ersten Mittelhandknochen.

Die Tunnelung sollte streng subkutan vorgenommen werden. Gerät man zu tief oder wird die Sehnenspannung zu stark, besteht die Gefahr der Kompressionsschädigung des N. ulnaris bis hin zur Ausbildung einer kompletten Parese.

Nachbehandlung/Prognose. Nach einer Opponensplastik wird in einer Unterarmdaumengipsschiene (EIP: Handgelenk in $20^{\circ}$ Extension, FDS IV: Handgelenk in $20^{\circ}$ Flexion) in voller palmarer $\mathrm{Ab}$ duktionsstellung für 3 Wochen ruhig gestellt, anschließend erfolgt die Krankengymnastik aus der Schiene heraus bei freigegebenem IP-Gelenk. Eine anfänglich behinderte radiale Abduktion verbessert sich meist im Lauf einiger Wochen.

Bei guten Voraussetzungen, korrekt durchgeführter Technik und einwandfrei- er Nachbehandlung ist die Prognose gut, wobei das Gesamtschädigungsbild häufig komplex ist, da es sich in vielen Fällen um Kombinationsverletzungen handelt [26].

\section{Ersatzoperationen nach sensiblem Ausfall des N. medianus}

Vom N. ulnaris oder N. radialis innervierte Hautareale der Hand werden gefäßgestielt (neurovaskulär) so umgelagert, dass die wichtigen medianusversorgten Kontaktzonen der Hand, nämlich die Beugeseite von Daumen und Zeigefinger, wieder für den Greifprozess eingesetzt werden können. Das operative Prinzip gleicht der sensiblen Rekonstruktion von Fingerdefekten durch gefäßgestielte Lappenplastiken: Als klassischer Lappen zur sensiblen Regeneration gilt der gestielte neurovaskuläre Insellappen nach Littler [15]. Er wird unter Einschluss des zugehörigen Gefäß-Nerven-Bündels von der Ulnarseite des Ringfingers gehoben. Die Beschaffenheit der Oberfläche und die sensible Versorgung der Hautinsel sind der Pulpa des Daumens sehr ähnlich. Nachteile dieser Lappenplastik ist die ausgedehnte Dissektion in der Hohlhand, die zu einer hohen Rate an Symptomen wie erhöhter Kälteempfindlichkeit oder Parästhesien im Spenderfinger führt.

Die Alternative stellt nach den Ergebnissen eigener Studien [27] der erstmals von Hilgenfeldt beschriebene, von Holevich verfeinerte und von Foucher u. Braun [11] zu einem echten Insellappen modifizierte neurokutane Lappen der ersten dorsalen Metakarpalarterie dar. Dieser ist neben der Arterie, an deren Begleitvenen und an einem sensiblen Endast des R. superficialis Nn. radialis gestielt. Seine Vorteile sind

- eine ähnliche Hautbeschaffenheit,

- eine technisch relativ einfache Lappenhebung und

- die variable Größe.

Der „Foucher-Lappen“ verfügt auch bei älteren Patienten über eine sehr gute sensible Qualität und kortikale Reorientierung [28], da keine Nervenkoaptation durchgeführt werden muss, und die Sensibilität somit sofort vorhanden ist. Auch hinterlässt der Hebedefekt kaum funktionelle Einschränkungen und ist ästhetisch 
akzeptabel. Der Lappen ist daher zur Rekonstruktion der Sensibilität am Daumen - nicht nur bei älteren Patienten - zu einem Verfahren der 1. Wahl geworden.

\section{Diskussion}

Optimierungen der mikrochirurgischen Nervenrekonstruktion wie verbessertes Instrumentarium, Einsatz intraoperativer Stimulation und „Faszikel-Mapping“, haben $\mathrm{zu}$ deutlichen Verbesserungen der funktionellen Resultate nach Nervenkoaptationen bzw. -rekonstruktionen geführt $[1,16,17]$. Dennoch verbleibt eine Anzahl von Defektheilungen nach peripheren Nervenverletzungen, bei denen motorische Ersatzoperationen indiziert sind, um eine Verbesserung der durch eine Lähmung eingeschränkten Handfunktion zu erreichen. Insbesondere bei Patienten jenseits des 45 . Lebensjahrs, aber auch bei jüngeren Menschen ist nach einer Nervenverletzung - auch bei optimaler Versorgung - nicht regelhaft mit einer Restitutio ad integrum zu rechnen. Gerade bei Verletzung von Stammnerven mit Ausfall motorischer Funktionen wird das Intervall zwischen Verletzung und Funktionsrückkehr vielfach bei der Therapieführung außer Acht gelassen. Einen Patienten mit zweifelhafter Prognose seinem Schicksal zu überlassen und auf den Ausgang der Nervenrekonstruktion zu warten, ist nicht mehr generell vertretbar. Zuwarten trotz bestehender Therapieoptionen kann für den Patienten nicht nur bleibende Behinderung, sondern auch die soziale Entwurzelung bedeuten. Noch immer werden leider in Plastisch/Handchirurgischen Kliniken viele Patienten mit Nervenausfällen vorgestellt, die bei früherer Behandlung durch eine Ersatzoperation längst wieder in ihr tägliches Leben integriert wären.

Die Gelenke der betroffenen Handund Fingerabschnitte sollten vor der Operation eine freie passive Beweglichkeit aufweisen. Die lokalen Weichteilverhältnisse in Hand- und Unterarmbereichen, die durch die Operation direkt betroffen sind oder durch welche die umgesetzten Sehnen verlaufen sollen, müssen geeignet sein. Fehlende Vernarbungen und ein ausreichendes Weichteilpolster für die umgesetzten Sehnen lassen ein opti- males Ergebnis erwarten. Auch sollte eine ausreichende Sensibilität, zumindest eine Schutzsensibilität, der Hand vorliegen.

Bei der synergistischen Verwendung von Muskeln, deren herkömmliche Funktion der neuen Funktion nach der Transposition weitgehend entspricht, ist eine schnelle Adaptation an die neue Muskelfunktion zu erwarten. Erstaunlich ist aber, dass auch bei Verwendung nicht synerger Muskeln sofort nach der Ruhigstellung eine zerebrale Adaptation festzustellen ist. Diese enorme Plastizität des Gehirns wird offensichtlich $u$. a. auch durch die Mikrobewegungen im Gips getriggert.

In die Überlegungen zur Operation sind teils konkurrierende, teils ergänzende Verfahren einzubeziehen, wie Arthrodesen und Tenodesen. Auch mikrochirurgische, neurovaskuläre Muskeltransplantationen bei Verlust ganzer Muskelgruppen sind heute möglich. Sie kommen v. a. bei ausgedehntem Muskeluntergang, wenn für eine Transposition geeignete Spendermuskeln fehlen, in Frage.

\section{Fazit}

Mit den heute zur Verfügung stehenden Optionen des motorischen und/oder sensiblen Ersatzes stehen Verfahren zur Verfügung, die für den Patienten nicht nur die Verbesserung der Handfunktionen, sondern v. a. auch eine Wiedererlangung verloren gegangener Lebensqualität bedeuten [10, 21, 23, 27]. Entscheidend für die Patienten ist dabei die enge interdisziplinäre Kooperation zwischen betreuendem Neurologen und Plastischem Chirurg. Dadurch können frühzeitig nach der erlittenen Verletzung der Status festgelegt und mögliche Therapieoptionen diskutiert werden. Hierbei ist v. a. auch durch eine sorgfältige Operationsplanung darauf zu achten, dass Ersatzoperationen nicht mit einer eventuellen Nervenregeneration, sei es spontan oder nach Rekonstruktion, interferieren, um dem Patienten alle Zukunftsoptionen zu erhalten und nicht unnötig „Brücken abzubrechen".

\section{Korrespondierender Autor}

\section{Prof. Dr. G. Germann}

Klinik für Hand-, Plastische und Rekonstruktive Chirurgie, Schwerbrandverletztenzentrum, BGUnfallklinik Ludwigshafen, Plastische und Handchirurgie, Universität Heidelberg

Ludwig-Guttmann-Straße 13,

67071 Ludwigshafen

guenter.germann@urz.uni-heidelberg.de

Interessenkonflikt. Es besteht kein Interessenkonflikt. Der korrespondierende Autor versichert, dass keine Verbindungen mit einer Firma, deren Produkt in dem Artikel genannt ist, oder einer Firma, die ein Konkurrenzprodukt vertreibt, bestehen. Die Präsentation des Themas ist unabhängig und die Darstellung der Inhalte produktneutral.

\section{Literatur}

1. Allan $\mathrm{CH}$ (2000) Functional results of primary nerve repair. Hand Clin 16:67-72

2. Boswick JA, Stromberg WB (1967) Isolated injury to the median nerve above the elbow. J Bone Joint Surg Am 49: 653-658

3. Bowden REM, Napier JR (1961) The assessment of hand function after peripheral nerve injuries. J Bone Joint Surg Br 43: 481-492

4. Brand PW, Beach RB, Thompson DE (1981) Relative tension and potential excursion of muscles in the forearm and hand. J Hand Surg [Am] 6: 209-219

5. Brandsma JW, Brand PW (1992) Claw-finger correction. Consideration in choice of technique. J Hand Surg [Br] 17: 615-621

6. Bruser $P$ (1997) Motor replacement operations in chronic ulnar nerve paralysis. Orthopäde 26: 690695

7. Bunnell S (1924) Reconstructive surgery of the hand. Surg Gynecol Obstet 39: 259-274

8. Burkhalter W, Christensen RC, Brown P (1973) Extensor indicis proprius opponensplasty. J Bone Joint Surg Am 55A: 725-732

9. Camitz H (1929) Über die Behandlung der Oppositionslähmung. Acta Chir Scand 65: 77-91

10. Chuinnard RG, Boyes JH, Stark HH et al. (1978) Tendon transfers for radial nerve palsy: use of superficialis tendons for digital extension. J Hand Surg [Am] 3: 560-570

11. Foucher G, Braun JB (1979) A new island flap transfer from the dorsum of the index to the thumb. Plast Reconstr Surg 63: 344

12. Hastings H 2nd, Davidson S (1988) Tendon transfers for ulnar nerve palsy. Evaluation of results and practical treatment considerations. Hand Clin 4: 167-178

13. Hoch J (1993) Correction of post-traumatic adduction insufficiency of the small finger by transposition of the tendon of the extensor indicis muscle. Handchir Mikrochir Plast Chir 25: 179-183

14. Jacobs B, Thompson TC (1960) Opposition of the thumb and its restoration. J Bone Joint Surg Am 42A: 1015-1026

15. Littler JW (1953) The neurovascular pedicle method of digital transposition for reconstruction of the thumb. Plast Reconstr Surg 12: 303

16. Lundborg G (2000) A 25-year perspective of peripheral nerve surgery: evolving neuroscientific concepts and clinical significance. J Hand Surg [Br] 25: 391-414 
17. Millesi $H$ (2000) Techniques for nerve grafting. Hand Clin 16: 73-91

18. Moberg E, Nachemson A (1967) Tendon transfers for defective long extensors of the wrist and fingers. Acta Chir Scand 133: 31-34

19. Omer GE Jr (1985) Reconstruction of a balanced thumb through tendon transfers. Clin Orthop 195: 104-116

20. Omer GE (1988) Timing of tendon transfers in peripheral nerve injury. Hand Clin 4:317-322

21. Raskin KB, Wilgis S (1995) Flexor carpi ulnaris transfer for radial nerve palsy: functional testing of long-term results. J Hand Surg [Am] 20: 737-742

22. Riordan DC (1969) Tendon transfers for median, ulnar or radial nerve palsy. Hand 1: 24-26

23. Skoll PJ, Hudson DA, De Jager W et al. (2000) Long term results of tendon transfers for radial nerve palsy in patients with limited rehabilitation. Ann Plast Surg 45: 122-126

24. Smith RJ (1986) Indications for tendon transfers to the hand. Hand Clin 2: 235-237

25. Thomsen M, Rasmussen KB (1969) Tendon transfers for defective long extensors of the wrist and fingers. Scand J Plast Reconstr Surg 3: 71-78

26. Toth S (1986) Therapist's management of tendon transfers. Hand Clin 2: 239-246

27. Traenkle M, Sauerbier M, Heitmann C et al. (2003) Restoration of thumb sensibility with the innervated first dorsal metacarpal artery island flap. J Hand Surg [Am] 5: 758-766

28. Traenkle M, Germann G, Heitmann C et al. (2004) Neurokutaner Insellappen nach Foucher. Chirurg 75: 996-1002

29. Trevett MC, Tuson C, De Jager LT et al. (1995) The functional results of ulnar nerve repair. Defining the indications for tendon transfer. J Hand Surg [Br] 20: 444-446

30. Zancolli EA (1957) Claw hand caused by paralysis of the intrinsic muscles. A simple surgical procedure for its correction. J Bone Joint Surg Am 39: 1076-1080 